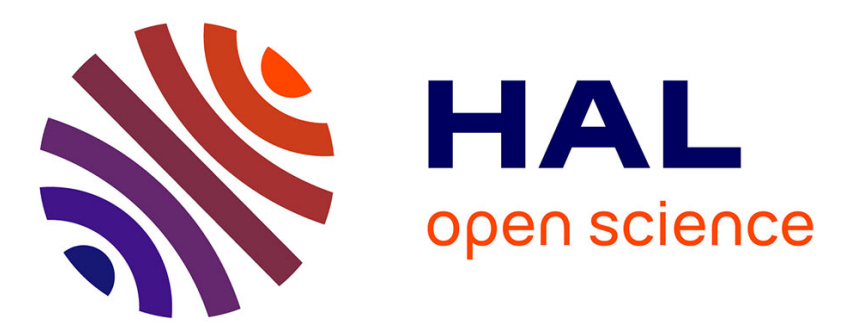

\title{
Quand le mandat s'étiole. Des cadres du ministère chargé des Sports saisis par l'affaiblissement et la mise en concurrence de leur activité professionnelle
}

\author{
Marina Honta, Samuel Julhe
}

\section{- To cite this version:}

Marina Honta, Samuel Julhe. Quand le mandat s'étiole. Des cadres du ministère chargé des Sports saisis par l'affaiblissement et la mise en concurrence de leur activité professionnelle. Sciences sociales et sport, 2016, 9, pp.115-152. halshs-01343982

\section{HAL Id: halshs-01343982 \\ https://shs.hal.science/halshs-01343982}

Submitted on 11 Jul 2016

HAL is a multi-disciplinary open access archive for the deposit and dissemination of scientific research documents, whether they are published or not. The documents may come from teaching and research institutions in France or abroad, or from public or private research centers.
L'archive ouverte pluridisciplinaire HAL, est destinée au dépôt et à la diffusion de documents scientifiques de niveau recherche, publiés ou non, émanant des établissements d'enseignement et de recherche français ou étrangers, des laboratoires publics ou privés. 


\section{QUAND LE MANDAT S'ÉTIOLE. DES CADRES DU MINISTĖRE} CHARGÉ DES SPORTS SAISIS PAR L'AFFAIBLISSEMENT ET LA MISE EN CONCURRENCE DE LEUR ACTIVITÉ PROFESSIONNELLE Marina Honta, Samuel Julhe

\section{L'Harmattan | « Sciences sociales et sport »}

2016/1 N 9 | pages 115 à 152

ISSN 1967-7359

ISBN 9782343093628

Article disponible en ligne à l'adresse :

http://www.cairn.info/revue-sciences-sociales-et-sport-2016-1-page-115.htm

\section{Pour citer cet article :}

Marina Honta, Samuel Julhe, "Quand le mandat s'étiole. Des cadres du ministère chargé des Sports saisis par l'affaiblissement et la mise en concurrence de leur activité professionnelle », Sciences sociales et sport 2016/1 ( ${ }^{\circ}$ 9), p. 115-152.

Distribution électronique Cairn.info pour L'Harmattan.

(C) L'Harmattan. Tous droits réservés pour tous pays.

La reproduction ou représentation de cet article, notamment par photocopie, n'est autorisée que dans les limites des conditions générales d'utilisation du site ou, le cas échéant, des conditions générales de la licence souscrite par votre établissement. Toute autre reproduction ou représentation, en tout ou partie, sous quelque forme et de quelque manière que ce soit, est interdite sauf accord préalable et écrit de l'éditeur, en dehors des cas prévus par la législation en vigueur en France. Il est précisé que son stockage dans une base de données est également interdit. 


\title{
Quand le mandat s'étiole. Des cadres du ministère chargé des Sports saisis par l'affaiblissement et la mise en concurrence de leur activité professionnelle
}

\author{
Marina HONT $A^{*}$ ET Samuel JULHE**
}

\section{Introduction}

L'action sportive de l'État français traduit, depuis le début de la $\mathrm{V}^{\mathrm{e}}$ République, l'attachement au principe de solidarité entre sport de masse et sport d'élite qu'organisent plusieurs dispositions législatives et réglementaires. Le ministère des Sports ${ }^{1}$ a, suivant cette logique, pris pour objet une pluralité de domaines, ce qui a eu pour corollaire de progressivement diversifier ses programmes d'intervention ${ }^{2}$ et les destinataires de l'action ${ }^{3}$. Pour élaborer et mettre en ouvre ces missions, le ministère mobilise des formes variées d'intervention: les orientations générales données aux établissements publics placés sous sa tutelle, l'animation du réseau de ses services déconcentrés et les conventions d'objectifs signées avec son partenaire historique, les fédérations spor-

\footnotetext{
Professeur des universités, université de Bordeaux. Chercheur Centre ÉmileDurkheim, UMR CNRS 5116, marina.honta@u-bordeaux.fr

** Maître de conférences des universités, université de Reims Champagne Ardenne. Chercheur au CEREP (EA 4692), samuel.julhe@u-bordeaux.fr.

${ }^{1}$ Le périmètre de ce ministère a régulièrement évolué ces dernières années et dépasse les questions sportives. Pour plus de clarté, nous avons ici réduit son appellation.

2 Organisation du sport de haut niveau, formations aux métiers du sport, soutien au développement du sport pour le plus grand nombre, contrôle et réglementation de la pratique, protection de la santé du sportif, lutte contre le dopage, la violence et les incivilités dans le sport, soutien à la construction d'équipements sportifs, aides à l'emploi, etc.

3 Le mouvement sportif, les collectivités territoriales et leurs groupements, divers « publics cibles ».
} 
tives. Il dispose par ailleurs d'agents relevant de corps propres ${ }^{4}$, tous de catégorie $\mathrm{A}$ ou $\mathrm{A}+$ : les inspecteurs de la jeunesse et des sports; les professeurs de sport regroupant les Conseillers en animation sportive (CAS) et les Conseillers techniques sportifs (CTS ${ }^{5}$; les Conseillers techniques et pédagogiques supérieurs (CTPS) ${ }^{6}$, ces deux derniers corps rassemblant plus généralement les Personnels techniques et pédagogiques (PTP) du secteur sport. Des agents administratifs ayant la particularité d'appartenir à des corps du ministère de l'Éducation nationale exercent également leur activité dans cette administration (les Ingénieurs et techniciens de recherche et de formation (ITRF), attachés, secrétaires administratifs, adjoints administratifs, techniciens ouvriers de service), cette situation s'expliquant par la proximité originelle de ces deux ministères.

Parmi l'ensemble de ces personnels, les PTP ont été chargés de conseiller et d'accompagner les dirigeants du mouvement sportif, les athlètes et les collectivités territoriales dans la mise en œuvre des orientations ministérielles, ce qui leur a permis d'acquérir une position interne et une visibilité sociale reconnues. Depuis une trentaine d'années toutefois, ce cadre institutionnel et professionnel a été traversé par divers changements. La pluralisation des acteurs mobilisés dans le secteur de l'organisation des activités physiques et sportives (APS) a progressivement instauré un environnement concurrentiel et, ce faisant, la nécessité pour les PTP de devoir composer avec une nouvelle division du travail. Les relations privilégiées, voire monopolistiques, qu'ils entretenaient avec les

\footnotetext{
${ }^{4}$ Les Conseillers d'éducation populaire et de jeunesse (CEPJ) en relèvent également, mais le propos est ici circonscrit au secteur sport.

${ }^{5}$ Décrets $n^{\circ} 85-720$ du 10 juillet 1985 et $n^{\circ} 2005-1718$ du 28 décembre 2005 relatifs à l'exercice des missions de conseillers techniques sportifs auprès des fédérations sportives. Antérieurement à cette date, les seuls corps propres du ministère étaient les inspecteurs généraux de la jeunesse et des sports et les inspecteurs principaux de la jeunesse, des sports et des loisirs alors régis par le décret $n^{\circ} 74-903$ du 25 octobre 1974 portant statuts particuliers des inspecteurs et inspecteurs principaux de la jeunesse, des sports et des loisirs.

${ }^{6}$ Décret $\mathrm{n}^{\circ} 2004-272$ du 24 mars 2004 relatif au statut particulier des conseillers techniques et pédagogiques supérieurs. Ce concours n'est actuellement ouvert qu'à l'interne et a essentiellement pour objet de permettre une mobilité statutaire aux professeurs de sport.
} 
athlètes, les dirigeants du mouvement sportif, les élus locaux, les stagiaires en formation, etc., sont ainsi devenues plus complexes à satisfaire et fidéliser. Parallèlement, les réformes administratives engagées par l'État pour s'adapter à ce pluralisme institutionnel et organisationnel touchant de très nombreux secteurs d'action publique comportent, elles aussi, des dispositions qui transforment le cadre de travail des PTP. Initialement expérimentaux et peu publicisés ${ }^{7}$, puis systématisés à partir de la loi organique relative aux lois de finances $(\mathrm{LOLF})^{8}$, ces programmes de modernisation à connotation néolibérale font de l'accroissement de la crédibilité de l'État par un meilleur pilotage de ses politiques publiques une composante majeure de ce processus?. Consacrant le passage d'une culture de moyens à une culture de résultats ou de performance, ils s'accompagnent également d'une compression des effectifs de fonctionnaires particulièrement significative avec la Révision générale des politiques publiques (RGPP) puis la Modernisation de l'action publique (MAP).

L'ensemble de ces dynamiques appelle ainsi à poursuivre l'analyse de ce que font les mutations de l'action publique aux professions liées à l'État, ces dernières, comme tout groupe professionnel, n'étant pas des entités stabilisées, mais confrontées en permanence aux changements ${ }^{10}$. L'approche de sociologie de l'action publique adoptée ici, parce qu'elle saisit l'action publique comme action politique - i.e. en tant que champ de divergences de normes substantielles - examine comment l'État et ses orientations gouvernementales (réformes, référentiels des politiques publiques, etc.) transforment les liens hiérarchiques existants, bouleversent les processus d'allocation des ressources, changent la nature des missions et des métiers, touchent aux identités professionnelles, affectent la répar-

\footnotetext{
${ }^{7}$ Bezes Philippe. 2009. Réinventer l'État, Paris, Presses de Sciences Po.

${ }^{8}$ Loi n ${ }^{\circ} 2001-692 \mathrm{du} 1^{\text {er }}$ août 2001.

${ }_{9}$ Dreyfus Françoise. 2010. «La révision générale des politiques publiques, une conception néolibérale du rôle de l'État ? " Revue française d'administration publique, vol. 136, $\mathrm{n}^{\circ} 4$, p. 857-864.

10 Demazière Didier; GadÉA Charles (dir.) 2009. Sociologie des groupes professionnels. Acquis récents et nouveaux défis, Paris, La Découverte; BOUSSARD Valérie ; DEMAZIÈRE Didier; Milburn Philip (dir.) 2010. L'injonction au professionnalisme. Analyse d'une dynamique plurielle, Rennes, PUR.
} 
tition des tâches et influent ainsi sur autant d'éléments qui forgent la légitimité professionnelle ${ }^{11}$.

Ainsi, et dans la mesure où chaque administration constitue un monde en soi avec ses logiques professionnelles, son rapport à l'État, son système de valeurs, ses métiers, ses formes de mobilité, ses modalités de promotion et ses relations interpersonnelles ${ }^{12}$, il est apparu opportun de cibler l'enquête sur les PTP ${ }^{13}$ (annexe 1). D'une part, car ils ont longtemps bénéficié d'un statut symbolique élevé, ce qui leur vaut d'être encore présentés comme « la force ${ }^{14} »$ du ministère des Sports. En outre, et alors qu'aucun projet professionnel précis ni idée de métier ne guide, le plus souvent, les futurs agents de plusieurs administrations ${ }^{15}$, les récits biographiques de ces professionnels révèlent qu'exercer dans le domaine du sport trouve ses racines dans un parcours entamé de façon précoce, durant l'enfance ou l'adolescence, avec un engagement très rapide dans une ou plusieurs disciplines sportives ${ }^{16}$. L'entrée dans le métier est le fruit d'un long processus d'incorporation d'un goût pour les APS, le plus souvent initié par le père et/ou un frère, et d'acculturation au monde sportif

11 Demailly Lise. 2008. «Introduction », in LE BIANIC Thomas; ViON Antoine (dir.). Action publique et légitimités professionnelles, Paris, LGDJ, p. 41 ; BEZES Philippe; DemaZiÈre Didier (dir.). 2011. «New Public Management et professions dans l'État: au-delà des oppositions, quelles recompositions », Sociologie du travail, $\mathrm{n}^{\circ}$ 53, p. 293-348.

${ }^{12}$ LINHART Danièle (coord.). 2006. Les différents visages de la modernisation du service public. Enquête sociologique sur les valeurs des agents de la fonction publique du Nord, Paris, La Documentation française ; AUST Jérôme; CRET Benoît. 2012. «L'État entre retrait et réinvestissement des territoires. Les délégués régionaux à la recherche et à la technologie face aux recompositions de l'action publique », Revue française de sociologie, vol. 53, $\mathrm{n}^{\circ} 1$, p. 3-33.

${ }^{13}$ Au nombre de 2640 au $1^{\text {er }}$ janvier 2012, BOUCHOUt Jean-Pierre ; JARRIGE Bertrand ; PRIMAUlT Jacques; WATRIN Daniel. 2012. Rapport relatif à la mission organisation ressources du ministère des Sports, de la Jeunesse, de l'Éducation populaire et de la Vie associative. Situation, diagnostic et scénarios, Inspection générale de la jeunesse et des sports, rapport 2012-M-10, 31 juillet, p. 33.

${ }^{14}$ C'est ainsi que la principale organisation syndicale représentant les intérêts des PTP, le Syndicat national des activités physiques et sportives (SNAPS, UNSA Éducation), conclut ses bulletins syndicaux (Flash Infos).

${ }^{15}$ LINHART D., op. cit.

${ }^{16}$ Les PTP exerçant les fonctions de CTS se distinguent généralement (mais pas systématiquement) des CAS par une spécialisation sportive plus forte. 
comme pratiquant-e, puis compétiteur ou compétitrice. Le réajustement d'une vocation pour les métiers de l'encadrement des APS, selon des mécanismes similaires au secteur de la culture ${ }^{17}$, parachève le processus. Dès la période du lycée, en effet, émerge le souhait de pouvoir développer et/ou encadrer cette ou ces APS à titre professionnel ou bénévole. Cette socialisation sportive précoce conduit également les interviewés à privilégier des formations dans le domaine sportif. Ces dernières et leur engagement associatif leur offrent alors l'opportunité de rencontrer, au cours des stages ou en marge de compétitions, des PTP et, ce faisant, de conforter leur choix professionnel en apprenant l'existence du concours du professorat de sport ${ }^{18}$. Leur goût des activités techniques et pédagogiques se renforçant avec le temps, l'exercice de ces métiers, que les personnes ont choisis et vivent sur le mode de la vocation, les conduit également à s'investir fortement ${ }^{19}$.

Dès lors, les changements internes et externes auxquels a été exposé le ministère des Sports sont appréhendés par les PTP comme menaçant aussi bien leur autonomie professionnelle que leur cœur de métier. Dans ce contexte, l'articulation entre deux formes de professionnalisme - celle étant le produit d'une définition interne et maittrisée par les travailleurs et celle portée par des acteurs extérieurs (hiérarchie, dirigeants sportifs, etc.) - est, ici aussi, rendue problématique, car elle confronte des intérêts, rationalités et normes en bien des points contradictoires ${ }^{20}$. Parce que les

\footnotetext{
${ }^{17}$ Dubois Vincent. 2013. La culture comme vocation, Paris, Raison d'agir.

${ }^{18}$ Ces professionnels, pour pouvoir y candidater, doivent être titulaires d'un diplôme de niveau II délivré soit par le ministère de l'Enseignement supérieur et de la Recherche (filière en sciences et techniques des activités physiques et sportives), soit par le ministère chargé des Sports : une licence en STAPS ou un Brevet d'État d'éducateur sportif (BEES) du second degré ou un Diplôme d'État supérieur de la jeunesse, de l'éducation populaire et du sport (DESJEPS). Outre ces aspects, des conditions spécifiques d'accès existent pour les sportifs de haut niveau ayant figuré pendant au moins trois ans sur la liste ministérielle (en catégorie élite, senior, jeune ou reconversion) et ayant suivi le cycle de formation à l'INSEP. Pour accéder à ce cycle de formation, les candidats doivent avoir réussi un examen probatoire.

${ }^{19}$ JulHe Samuel ; HonTA Marina. 2015. «Expression et maintien de la passion au travail chez les agents du ministère des Sports », in LORIOL Marc ; LEROUX Nathalie (dir.). Le travail passionné. L'engagement artistique, sportif ou politique, Toulouse, Érès.

${ }^{20}$ Boussard V. ; Demazière D. ; Milburn P., op. cit., p. 19.
} 
formes d'adaptation ou de résistance qu'ils déploient visent avant tout à rappeler et à préserver les référentiels professionnels antérieurement acquis, il en ressort l'image passablement brouillée d'un groupe qui continue de défendre une identité corporatiste traditionnelle, mais dont les membres sont de plus en plus enclins à l'individualisme.

\section{I- Des PTP exposés aux reconfigurations de leur environnement de travail}

Bien que le ministère des Sports demeure actif, son action doit désormais être analysée en la replaçant dans une configuration plus large d'acteurs tant publics que privés (collectivités territoriales, fédérations, entreprises) qui a transformé l'environnement de travail des PTP et par là même déstabilisé leur position acquise dans le champ de l'organisation des APS. Le tournant néo-managérial par ailleurs, consacré plus particulièrement par les effets puissants de la RGPP, accentue les ajustements nécessaires.

\section{a) Le ministère des Sports : une administration progressivement concurrencée et contestée}

Le système français d'organisation du sport construit à partir de la fin de la Seconde Guerre mondiale repose sur un partenariat, longtemps singulier en Europe, entre le ministère des Sports et le mouvement sportif. Aussi, le modèle du «corporatisme sectoriel» ou de «l'étatisme», convoqué, notamment, pour analyser les interactions entre l'État et les groupes d'intérêt dans le secteur de l'agriculture ${ }^{21}$, renvoie-t-il également aux modes de relations rencontrés dans le champ sportif ${ }^{22}$. En ce sens, par la procédure de délivrance de l'agrément et de la délégation aux fédérations sportives faisant suite à leur adoption de dispositions conformes à des orientations obligatoires, l'État leur confère la responsabilité de gérer une ou plusieurs disciplines sportives. Ces principes, fixés sur les

\footnotetext{
${ }^{21}$ Jobert Bruno; MULLER Pierre. 1987. L'État en action : politiques publiques et corporatismes, Paris, PUF. Muller Pierre. 1984. Le technocrate et le paysan, Paris, Éditions Économie et Humanisme - Les Éditions Ouvrières.

${ }^{22}$ HonTA Marina. 2010. Gouverner le sport. Action publique et territoires, Grenoble, PUG.
} 
plans législatif et réglementaire, prévoient également que les fédérations sont placées sous la tutelle du ministère qui conserve un droit de regard sur leurs activités. Les axes du partenariat construit entre ces deux catégories d'acteurs visent à maintenir, voire à améliorer le rang de la France dans le classement des meilleures nations sportives. Ils ont également pour objet le développement d'une pratique sportive de qualité et diversifiée pour le plus grand nombre sans recherche systématique de performance. Responsables de la mise en œuvre de ces orientations, les fédérations sportives reçoivent un soutien économique et en personnels de l'État.

S'agissant du premier type d'aide, participant plus généralement à la promotion du mouvement sportif, les crédits ministériels que les fédérations se voient attribuer sont complétés par ceux du Centre national pour le développement du sport (CNDS) venant en soutien au Comité national olympique et sportif français (CNOSF) et aux diverses associations sportives locales mobilisées dans la promotion du sport pour le plus grand nombre à des fins de réduction des inégalités sociales et territoriales d'accès à la pratique. Au titre des aides en personnels, ce sont les CTS, "placés auprès » des fédérations ou de leurs instances territoriales (les ligues ou comités régionaux), qui représentent, depuis la fin des années 1950 d'abord en tant que cadres techniques et pédagogiques (CTP), une ressource afin qu'elles mettent en œuvre ces missions ${ }^{23}$. Ils occupent ainsi une position centrale dans le partenariat entre le mouvement sportif et le ministère.

Ces axes au périmètre très étendu font que les PTP apparaissent, dans cette administration également, comme un groupe-pivot ${ }^{24}$ : chargés de conseiller et d'accompagner, plus particulièrement le mouvement sportif, dans la mise en œuvre de ces orientations, ils ont acquis une position interne et une visibilité sociale fortes et reconnues, symbolisant en outre le volontarisme de l'État-providence dans ce secteur. Sans leur mobilisation au travail, en effet, l'objectif du développement des associations et

\footnotetext{
${ }^{23}$ Décret n ${ }^{\circ}$ 63-435 du 29 avril 1963 portant statut du personnel contractuel des cadres techniques et pédagogiques de la jeunesse et des sports.

${ }^{24}$ Boussard V. ; Demazière D. ; Milburn P., op. cit., p. 25.
} 
des pratiquants sportifs fixé par le ministère des Sports n'aurait pu être atteint.

Depuis les années 1980, plusieurs facteurs ont progressivement participé de l'étiolement de leur mandat, i. e. des actions qui ne sont ni permises aux autres ni attendues des autres ${ }^{25}$ et, ce faisant, du déclin de leur prestige.

En premier lieu, le secteur de l'organisation du sport n'échappe pas à la pluralisation des acteurs mobilisés. Qu'il s'agisse des entreprises ou des collectivités territoriales et de leurs groupements, leurs formes d'engagement sont désormais plurielles et contribuent indéniablement à la promotion tant de l'excellence sportive que du sport pour le plus grand nombre. Témoignant de l'avènement d'une économie internationalisée de biens et de services, la mobilisation des premières se manifeste par des formes variées d'intervention : prestations de services sportifs, sponsoring de clubs et d'athlètes, mécénat, commercialisation de produits dérivés, médiatisation des spectacles sportifs, organisation d'événements, etc. La participation croissante de ces acteurs a été profitable aux fédérations sportives, principalement à celles organisant les disciplines les plus médiatisées ${ }^{26}$. Cet accroissement de ressources a ainsi permis à plusieurs d'entre elles de réduire leur dépendance à l'État tant sur le plan financier qu'à l'égard des aides en personnels. Il suscite en outre des velléités croissantes d'autonomisation de la part de plusieurs dirigeants sportifs qui ont progressivement remis en cause la nature tutélaire des rapports historiquement construits avec le ministère des Sports. Aussi, les présidents du CNOSF et de plusieurs fédérations sportives revendiquent-ils désormais un autre modèle de gouvernance du sport, modèle

\footnotetext{
${ }^{25}$ Hugues Everett. C. 1996. Le regard sociologique. Essais choisis, Paris, EHESS.

${ }^{26}$ COUR DES COMPTES. 2009. L'État et les fédérations sportives face aux mutations du sport, rapport d'activité, p. 488-489. Rappelant que les fédérations sportives disposent de trois ressources principales: les subventions publiques, les ressources statutaires (produits des licences en particulier...) et les recettes commerciales, issues de la billetterie des compétitions qu'elles organisent, de la cession des droits audiovisuels et des droits de marketing dérivés, la part relative de ces ressources au sein du budget des fédérations que la Cour a contrôlées varie significativement : les ressources non commerciales sont légèrement majoritaires dans trois fédérations (athlétisme, basket-ball, handball) et marginales dans les deux autres (football et rugby).
} 
dans lequel l'État ne disposerait plus de «tous les leviers de commande ${ }^{27}$ ", mais aussi le fait de se voir confier la responsabilité de missions actuellement partagées comme le développement du sport de haut niveau ${ }^{28}$ ou la formation ${ }^{29}$.

La décentralisation, ensuite, a profondément transformé le paysage institutionnel, bien que l'État n'ait pas transféré de compétence aux collectivités territoriales dans ce secteur d'action publique ${ }^{30}$. Ayant saisi l'opportunité offerte par la clause générale de compétences, plus de 30 ans après la réforme, leur volontarisme en matière de promotion du sport pour le plus grand nombre et de l'excellence sportive est régulièrement démontré $^{31}$. Elles contribuent à $30 \%$ de la dépense sportive, alors que la part de l'État (incluant les crédits du ministère de l'Éducation nationale comprenant le traitement des professeurs d'éducation physique et sportive) est de $12 \%$. Les communes représentent ici les principaux partenaires des associations sportives ${ }^{32}$. Les initiatives du ministère des Sports visant à composer avec cette nouvelle configuration territoriale n'ont pas manqué (renforcement des prérogatives des services déconcentrés en matière d'expertise, notamment afin d'accompagner les élus locaux dans l'élaboration des politiques sportives territoriales), mais malgré celles-ci, les missions ministérielles se révèlent complexes à assumer et valoriser face à des collectivités s'émancipant, voire concurrençant le ministère en matière sportive. Les rapports de compétition territoriale marqués par des volontés d'hégémonie peuvent effectivement être nom-

\footnotetext{
${ }^{27}$ MASSEgLiA Denis. 2010. «L'État ne doit plus décider seul ! », Acteurs du sport, n 123 , p. 18.

${ }^{28}$ Comité national olympique et sportif français. 2014. Projet du CNOSF pour le sport français. Une ambition : "Passer d'une nation de sportifs à une nation sportive». Une clé : «Rénover le modèle sportif français », 9 janvier (www.franceolympique.com).

29 PIERRE Jérémy. 2013. "Vers une nouvelle gouvernance des conditions d'accès à l'encadrement sportif professionnel », Staps, vol. 1, n 99, p. 81-92.

30 À l'exception de la constitution des Commissions départementales des espaces, sites et itinéraires (CDESI) de sports de nature pour les conseils départementaux.

31 Honta Marina. 2014. «Sport et action publique locale ou cette réforme dont personne ne veut», in CHARRIER Dominique ; LAPEYRONIE Bruno, Les politiques sportives territoriales. Savoirs et questionnements, Dardilly, Kreaten, p. 243-253.

32 Megherbi Damien. 2013. «Le poids économique du sport en 2010 », Stat-Info, $\mathrm{n}^{\circ} 13-01$, mars.
} 
breux sur certains territoires ${ }^{33}$. Disposant, elles-mêmes en interne, des compétences de professionnels relevant de la fonction publique territoriale $^{34}$, elles s'affranchissent, au moins en milieux urbain et péri-urbain, de l'expertise des PTP, plus particulièrement de celle des CAS et CTPS exerçant dans les services déconcentrés. À tous ces titres, elles revendiquent désormais, par la mobilisation notamment de l'Association nationale des élus en charge du sport (ANDES), que leur rôle soit mieux reconnu. Leur implication croissante a ainsi, et dans ce secteur également, participé au mouvement de marginalisation des services déconcentrés du ministère des Sports que les réformes néo-managériales du début des années 2000 ont, par la suite, accentué.

Plus généralement, la multiplication de ces intervenants a affaibli cette administration qui n'a plus la même légitimité, les nombreuses successions de ministres à sa tête depuis 2007 ajoutant par ailleurs à ce constat. Aux changements exogènes exposés ici et pouvant désormais transformer les partenaires traditionnels de cette administration en rivaux, en raison de l'accroissement de leurs ressources et influence, s'additionnent en outre des changements internes.

\section{b) Des configurations de travail transformées}

Les réformes dont a fait l'objet le ministère des Sports se sont accélérées ces dernières années. Largement inspiré des préceptes issus du secteur privé et porté par le New Public Management (NPM), le processus engagé instaure un important resserrement des missions et de l'organisation du réseau des établissements publics, une restructuration des services déconcentrés et une réduction des ressources, notamment des effectifs de PTP ${ }^{35}$.

\footnotetext{
${ }^{33}$ HontA Marina. 2011. «L'État, le marché et les pratiques locales : la problématique gouvernance de l'excellence sportive en France », Politique et Sociétés, vol. 30, n 3, p. 75 98.

${ }^{34}$ La filière sportive a été créée en 1992.

35 HonTA Marina. 2009. "Révision générale des politiques publiques et réforme du ministère de la Santé et des Sports : la justification par la performance », Revue juridique et économique du sport, $\mathrm{n}^{\circ}$ 91, p. 7-25.
} 
S'agissant du premier point, les transformations, présentées notamment en comité technique paritaire ministériel ${ }^{36}$, prévoient le renforcement de la tutelle des établissements par la Direction des sports du ministère et le recentrage de leurs missions sur les priorités nationales définies au travers du Projet annuel de performance (PAP) : l'accueil des sportifs de haut niveau afin de permettre la réussite de leur double projet; l'offre de formation aux diplômes conduisant aux métiers de l'animation et du sport dans le secteur monopolistique, i. e. des activités se déroulant dans un environnement spécifique (plongée en scaphandre et en apnée, en milieu naturel et en fosse de plongée; canoë-kayak en rivière de classe supérieure à trois ; voile au-delà de 200 milles nautiques d'un abri ; canyonisme; parachutisme ; ski et alpinisme; spéléologie ; surf de mer; vol libre), et dans les secteurs où l'offre de formation privée (associative et marchande) est encore insuffisante. La réforme a également abouti à un resserrement du nombre de Centres de ressources, d'expertise et de performance sportive (CREPS) avec la fermeture de huit d'entre eux. Ces décisions, prises rapidement après la réalisation d'audits et la remise au président de la République du premier rapport d'étape sur la RGPP par Éric Woerth ${ }^{37}$, ont alors suscité la mobilisation des organisations syndicales et de plusieurs agents du ministère afin de contester, en vain, la fermeture de ces établissements et leurs incidences sur leurs missions et agents (mobilité géographique forcée pour les agents titulaires, fin de mission pour les contractuels) ${ }^{38}$.

\footnotetext{
${ }^{36}$ Ministère de la Santé, de la Jeunesse, des Sports et de la Vie associative. Secrétariat d'État aux sports, à la jeunesse et à la vie associative, La refonte du réseau des établissements publics nationaux du secteur "sport et formations 》, 16 décembre 2008.

${ }^{37}$ Ce rapport (3 décembre 2008) centré sur l'avancement des réformes RGPP dans chaque ministère octroie "un feu rouge » (attestant donc d'un retard au regard de la période fixée : avril 2008) au ministère des Sports sur ce chapitre de la «Redéfinition des missions des CREPS, évaluation de ces établissements et sur cette base suppression éventuelle d'une partie d'entre eux ». Le second rapport d'étape (13 mai 2009), suite aux décisions annoncées par B. Laporte alors secrétaire d'État aux Sports, affiche lui tous les signaux « au vert».

${ }^{38}$ Le titre du quotidien L'Humanité du 16 décembre 2008, jour de cette mobilisation, est particulièrement évocateur, aussi bien des annonces qui étaient attendues que des inquiétudes des personnels : «Le ministère sur le grill ». Lire aussi l'analyse du SEJS,
} 
Concernant le second point, les décisions gouvernementales de réorganisation des Directions régionales et départementales de la jeunesse et des sports (DRJS et DDJS) font plus généralement écho à la progressive «résidualisation» de ces services depuis la décentralisation ${ }^{39}$. Ainsi, et devant le problème également posé par la taille critique de certaines DDJS, Roselyne Bachelot-Narquin a annoncé, dès sa prise de fonction en tant que ministre de la Santé et des Sports en juin 2007, des rapprochements entre celles-ci et les Directions départementales des affaires sanitaires et sociales (DDASS), mais aussi entre les DRJS et les Directions régionales des affaires sanitaires et sociales (DRASS). Le lancement de la Réforme de l'administration territoriale de l'État (RéATE) entérine ce processus ${ }^{40}$. Élaboré par plusieurs textes entre les mois de juillet 2007 et 2008 , le cadre de cette réforme repose sur divers principes ${ }^{41}$ : le renforcement de l'échelon régional et l'affirmation de l'autorité préfectorale sur des services déconcentrés fusionnés afin de mieux piloter l'action interministérielle de l'État. À l'échelle départementale, le principe retenu est celui de directions interministérielles (DDI) sans lien organique direct avec les ministères, car elles sont placées sous l'autorité du préfet et gérées directement par le secrétariat général du gouvernement. Les anciens services déconcentrés du ministère des Sports ont, dans ce contexte, connu une double intégration. Dans le champ des affaires sociales d'une part; dans le cadre de nouvelles directions relevant de plusieurs ministères ou du Premier ministre créées par la RéATE : les Directions régionales de la jeunesse, des sports et de la cohésion sociale (DRJSCS) et les Directions départementales de la cohésion sociale ou de la Cohésion so-

Conséquences de la révision générale des politiques publiques (RGPP) pour l'avenir du secteur de la Jeunesse et des Sports, 17 avril 2009.

${ }^{39}$ EPSTEIN Renaud. 2013. La rénovation urbaine. Démolition-reconstruction de l'État, Paris, Presses de Sciences Po, p. 226.

${ }^{40}$ Instruction $\mathrm{n}^{\circ}$ 5285/SG du 19 mars 2008 relative à la réforme de l'organisation des services territoriaux de l'État et circulaires n 5316/SG du 7 juillet 2008 et n ${ }^{\circ}$ 5359/SG du 31 décembre 2008 relative à l'organisation de l'administration départementale de l'État.

${ }^{41}$ Peneau Valérie et al., 2012. Réforme de l'administration territoriale de l'État. Optimisation des modalités de gestion budgétaire et de gestion des ressources bumaines, Inspection générale des finances, rapport n 2012-M-009-01. 
ciale et de la protection des populations (DDCS/DDCSPP). Chronologiquement, la décision de rapprocher les services afin de favoriser une nouvelle interministérialité nécessaire au renforcement de l'unité d'action de l'État n'a pas été précédée de la redéfinition de leurs missions ${ }^{42}$. Or, les textes qui ont suivi ont sensiblement reconduit les missions des exDDJS et DRJS alors que s'imposait, dans le même temps, la nécessité pour le ministère des Sports de faire face à la réduction continue des effectifs et des moyens, celle-ci s'étant accélérée d'abord avec la RGPP puis la MAP.

Les politiques de réduction des emplois publics ont effectivement conduit les ministres successifs à d'abord faire porter les suppressions sur les effectifs des personnels administratifs, effectifs longtemps restés stables, afin de préserver «le cœur de métier» représenté par les agents des corps propres ${ }^{43}$. À partir des années 2008-2010, les marges de suppressions d'emplois dans les fonctions supports étant épuisées, la fermeture de plusieurs CREPS a permis « de rendre des emplois » supplémentaires dans ce secteur, mais dans des proportions n'étant pas à la mesure des contraintes. Des arbitrages ont alors été nécessaires afin de répartir et programmer, sur la période du budget triennal (2010-2012), les suppressions au sein même des corps propres du ministère. S'il est complexe d'identifier précisément ces pertes ${ }^{44}$, le corps des Inspecteurs jeunesse et sports et la fraction des CAS de celui des professeurs de sports exerçant dans les services déconcentrés et les établissements ont été plus particulièrement concernés. Les effets de la «sanctuarisation ${ }^{45}$ » des emplois de

${ }^{42}$ KADA Nicolas. 2012, « La réforme de l'État territorial », Revue française d'administration publique, vol. 1, n 141, p. 109-120.

${ }^{43}$ Bouchout J.-P. ; Jarrige B. ; Primault J. ; Watrin D. ; op. cit., p. 16.

${ }^{44}$ D’une part, car la LOLF a transformé les « emplois budgétaires » référencés par catégories et par corps jusqu'en 2005 en «nombre d'ETPT» indifférencié et en «masse budgétaire ». D’autre part, car les crédits de rémunération des effectifs propres au ministère figuraient jusqu'en 2010 dans un programme spécifique (210) «conduite et pilotage » au sein de la mission «sport, jeunesse et vie associative » alors qu'aujourd'hui ils ont été transférés dans le programme 124 de la mission "solidarité, insertion et égalité des chances » justifié par une volonté de simplification, mais qui ne permet plus l'identification ex ante des ressources humaines disponibles par secteur (idem, p. 27)

45 D'ETTORE Gilles. 2012. Rapport d'information sur la gouvernance des fédérations sportives, enregistré à la présidence de l'Assemblée nationale le 22 février. 
CTS, dans la perspective de la préparation des JO de 2012, les a en effet protégés des mesures de non-remplacement d'un fonctionnaire sur deux partant à la retraite et expliquent ainsi que ce segment des PTP a pu apparaitre, au moins dans un premier temps, comme le " grand gagnant » de la réforme (tableau 1).

\section{Tableau 1}

Comparatif des effectifs physiques de PTP entre 2009 et $2012^{46}$

\begin{tabular}{|c|c|c|}
\hline Affectations & $\mathbf{1}^{\text {er }} \mathbf{0 9 / 2 0 0 9}$ & $\mathbf{1}^{\text {er }} \mathbf{0 1 / 2 0 1 2}$ \\
\hline $\begin{array}{c}\text { Services déconcentrés } \\
\text { (CAS) }\end{array}$ & 950 & 797 \\
\hline Établissements publics & $\begin{array}{c}450 \text { formateurs } \\
\text { (sport) }\end{array}$ & $\begin{array}{c}305 \text { formateurs } \\
\text { (sport/jeunesse et } \\
\text { éducation populaire) }\end{array}$ \\
\hline $\begin{array}{c}\text { Placés auprès du } \\
\text { mouvement sportif (CTS) }\end{array}$ & 1670 & 1684 \\
\hline
\end{tabular}

Si la MAP doit permettre une gestion plus différenciée de la baisse des effectifs calculée désormais sur d'autres bases (- 2,5\% des effectifs par an), ces réductions, s'agissant du ministère des Sports, ont été reconduites pour le nouveau triennal budgétaire (2013-2015) afin de compenser les créations d'emplois dans les secteurs jugés " prioritaires » (éducation, justice, sécurité).

Ces éléments, indispensables pour appréhender les jeux de pouvoir dans lesquels se trouvent pris les PTP ainsi que leur contexte spécifique de travail, représentent des mutations qui menacent tout autant leur autonomie professionnelle que les missions qui fondent leur cœur de métier. Dans cette configuration, la production de rhétoriques et pratiques défensives et le rappel constant des systèmes de référence antérieurs figurent au titre des stratégies visant à résister à ces mutations.

\footnotetext{
${ }^{46}$ Bouchout J.-P. ; Jarrige B. ; Primault J. ; Watrin D., op. cit., p. 33.
} 


\section{II- Une standardisation des pratiques mettant à mal le modèle professionnel défendu par les PTP}

Le travail des PTP, au plus près des représentants des organisations sportives et des athlètes, s'est longtemps organisé loin de la surveillance de la hiérarchie ministérielle. Ceci a ouvert un espace substantiel d'autonomie à ces « travailleurs itinérants » consacré par ailleurs réglementairement. L'arrêté du 28 décembre 2001 prévoit ainsi que les PTP relèvent de l'article 10 du décret du 25 août 2000 et qu'à ce titre, ils bénéficient d'une large autonomie dans l'organisation de leur activité ${ }^{47}$. Ce faisant, ils ne sont pas soumis à un décompte horaire du temps de travail. Les changements issus des diverses réformes administratives ont eu pour effet de transformer aussi bien le cadre que les méthodes d'organisation du travail. Les PTP ont eux aussi été exposés à une logique externe, imposée notamment par l'État, logique pouvant être qualifiée de bureaucratique ou managériale en ce qu'elle induit un contrôle de leur activité par un corps administratif ${ }^{48}$. Dès lors, le processus de réorganisation des services, les effets délétères de la réduction des effectifs et l'introduction d'outils de suivi des activités professionnelles ont-ils accentué, ici aussi, la tendance à la standardisation des pratiques de travail ${ }^{49}$. Interférant avec les référentiels professionnels valorisés par les PTP, une telle dynamique déstabilise ce groupe et renforce sa segmentation.

\footnotetext{
47 Décret $n^{\circ} 2000-815$ du 25 août 2000 relatif à l'aménagement et à la réduction du temps de travail dans la fonction publique de l'État et dans la magistrature : «Le régime de travail de personnels chargés soit de fonctions d'encadrement, soit de fonctions de conception lorsqu'ils bénéficient d'une large autonomie dans l'organisation de leur travail ou sont soumis à de fréquents déplacements de longue durée peut, le cas échéant, faire l'objet de dispositions spécifiques adaptées à la nature et à l'organisation du service ainsi qu'au contenu des missions de ces personnels. »

48 BOUSSARD Valérie. 2009. «Les policiers de la sécurité publique et l'obligation de faire du chiffre », in Boussard V. ; DemaziÈre D. ; Milburn P., op. cit., p. 111.

${ }^{49}$ BelorgeY, Nicolas. 2011. "Réduire le temps d'attente et de passage aux urgences. Une entreprise de "réforme" d'un service public et ses effets sociaux", Actes de la recherche en sciences sociales, vol. $4, \mathrm{n}^{\circ} 189$, p. 16-33.
} 
Sciences sociales et sport, $n^{\circ}$ 9, 2016, p. 115-152

\section{a) L'accroissement de sources normatives exogènes : des marges d'autonomie rognées}

Si la RéATE avait pour objet d'opérer une simplification de l'organisation administrative, sa mise en œuvre a donné lieu à des modalités n'ayant pas toutes fait la preuve de leur pertinence. À ce titre, la Cour des comptes $^{50}$ distingue-t-elle les directions régionales qualifiées de «bien assises » de celles, comme les DRJSCS, qui «posent davantage de problèmes ». Elle souligne également que les DDCS et DDCSPP, plus particulièrement, sont confrontées à de multiples difficultés que les PTP rencontrés ont largement exprimées.

Ils évoquent ainsi le fait que leurs pratiques de travail (travail par objectifs, dimension éducative des missions, horaires atypiques et nombreux déplacements sur le terrain), parce qu'elles se distinguent fortement de celles des agents des ex-DRASS et ex-DDASS qui relèvent de logiques d'organisation plus traditionnelles d'une administration de gestion (présence imposée dans les bureaux, respect d'horaires «classiques » dans l'administration), ont fait l'objet de très nombreuses incompréhensions tant de la part de leurs (nouveaux) collègues de travail que des équipes de direction lorsque les membres provenaient du secteur des affaires sociales :

«Ce que l'on entend dans les DD et c'est pareil pour les autres CAS que je rencontre, c'est "restez dans votre bureau pour accueillir le public. Par rapport aux autres dans la recomposition, les autres il faut qu'ils voient que vous êtes dans votre bureau". Et ça, quand on a passé un concours où on est plus sur l'accompagnement des personnes... tu peux toujours attendre que les gens viennent. J'ai fait des tests certains jours à attendre derrière mon ordinateur! Il y a une forme d'incompréhension de notre fonction, une forme de dénigrement à chaque fois qu'on n'est pas au bureau... Être regardé comme des branleurs, ça c'est difficile. Moi je l'ai vécu quand j'allais travailler avec le CROS ou le CDOS. Si les gens nous voient arriver au bureau et qu'ils ne nous ont pas vus depuis deux jours alors là... et puis des

\footnotetext{
${ }^{50}$ COUR DES COMPTES. 2013. L'organisation territoriale de l'État, rapport public thématique.
} 
questions quoi?! "Ah c'est maintenant qu'on arrive au bureau? !" Cette relation-là c'est très pernicieux, on subit une incompréhension, l'état des lieux est catastrophique. L'administration s'est transformée en ce qu'elle a de pire !» (Un CAS en DDCS, 15 ans d'ancienneté)

De telles différences de cultures professionnelles ont rapidement suscité des velléités d'homogénéisation des conditions de travail (fixation de plages au cours desquelles la présence de la totalité du personnel est obligatoire, nécessité de "pointer») de la part des chefs de service afin de mettre fin aux incompréhensions, jalousies ou stigmatisations entre agents. Au-delà du climat social néfaste qui a pu s'installer dans les directions, les PTP contestent vivement ces injonctions normatives et ces glissements, vécus comme des sanctions, entre deux situations professionnelles : celle qu'ils estiment devoir rester la leur et qui symbolise l'autoorganisation et l'autonomie et celle d'agents administratifs confrontés à une logique bureaucratique dépourvue d'intérêt et disqualifiant leur fonction :

«Le mal-être est fort parce que les gens sont sur des logiques professionnelles différentes. Les PTP ne se retrouvent plus dans leur métier, ils ne peuvent plus avancer comme ils ont envie d'avancer et tu le sens sur les discussions des fois, dans des situations... Les réunions de service servent à régler les problèmes, mais des problèmes de pointeuse parce qu'il y a le système de la pointeuse qui est venu... Ils me demandaient mon avis là-dessus, je leur ai dit: "Mais il n'y a pas de souci, je peux pointer. La seule chose c'est que le jour où j'arrive à 1607 heures, j'arrête. Donc, si c'est au $1^{\text {er }}$ octobre, le $1^{\text {er }}$ octobre, j'arrête et je reviens le 2 janvier, mais vous prenez tout en compte". Et puis cette logique, après, de méconnaissance des missions de chacun. Nous, on est toujours vus comme des extraterrestres. On n'a pas d'horaires typiques parce qu'ils ne comprennent pas que des fois tu vas partir à $3 \mathrm{~h}$ de l'après-midi parce que tu as rendez-vous à l'extérieur. » (Un CAS en DRJSCS, 14 ans d'ancienneté)

Acculés à un travail de justification permanente, certains PTP refusent donc explicitement de se conformer à ces exigences externes en invoquant le fait qu'elles vont à l'encontre du cadre réglementaire organisant 
leur travail. Ce faisant, elles sont vécues comme menaçant leur professionnalité définie par opposition à celles des autres fonctionnaires contraints par des logiques bureaucratiques. Elles sont en outre perçues comme un affaiblissement du service public, car elles ont fortement influé sur la fréquence, voire l'existence même des relations entre les PTP et les acteurs locaux, principalement les partenaires associatifs. Tous - et pas seulement les plus anciens dans la profession qui assumaient déjà il y a plusieurs années les fonctions de conseiller de secteur les amenant à aller rencontrer régulièrement les acteurs sportifs - évoquent désormais la transformation de leur activité et la «rupture » consacrée par ces reconfigurations :

«La perte d'identité, ça a été vraiment ça... Le fait déjà qu'on ne s'appelle pas Jeunesse et Sports, enfin en tout cas pour nous. Je vois des structures qui ne savaient pas que Jeunesse et Sport existe encore. Enfin qu'on s'occupe encore du sport et on voit de moins en moins d'usagers. En termes de conseils aux associations, par exemple sur le CNDS, les gens n'ont pas le réflexe de venir voir le conseiller. Ils viennent le voir pour rendre le dossier et puis signer et c'est tout. Mais ils ne savent pas que le conseiller peut être là pour les aider en amont du dossier. Il y a toute cette démarche et puis les gens ne savent plus du tout ce qu'on fait parce qu'ils entendent DDCSPP. Déjà rien que le nom, quand tu viens en assemblée générale, en règle générale, ça met un peu de sourires à tout le monde. Tu es obligé d'expliquer. Quand tu es avec les services vétérinaires, avec la répression des fraudes, ils sont là à nous dire "mais vous faites quoi ?" » (Un CAS en DDCSPP, 4 ans d'ancienneté)

Cette perte d'autonomie dans la possibilité d'organiser leur travail n'est pas exprimée par les seuls CAS. Bien qu'ils constituent un segment des PTP qui a eu du mal à se situer par rapport au champ de la réorganisation territoriale de l'État, car ils exercent leurs missions auprès des fédérations, les CTS déplorent eux aussi le déploiement d'initiatives visant le contrôle de leur activité par l'autorité administrative. La direction des sports en effet, afin de mieux assurer le suivi de leurs missions, a adopté l'outil de gestion qu'est le «CTS Web». Consistant à ce qu'ils renseignent hebdomadairement le temps respectivement consa- 
cré à leurs différentes tâches, ce dispositif est présenté par l'administration centrale comme nécessaire à la formalisation et au repérage de leurs modes d'organisation du travail ${ }^{51}$. Il s'agit là d'une évolution que les CTS interprètent comme un mouvement de disciplinarisation menaçant directement leur l'autonomie professionnelle. Ils se montrent ainsi très réservés, voire réticents, vis-à-vis de cette initiative, réticence relayée en outre par le SNAPS ${ }^{52}$. Malgré cela, quelques-uns y voient l'opportunité de faire remonter auprès de l'administration centrale des informations sur l'exercice concret des missions :

«Cela a été mal accueilli je pense parce que c'est du flicage, c'est sûr... Après, on en a discuté, on s'est dit : "bon, il doit y avoir quand même des avantages à cet outil”. Et au final, on y voit un intérêt. Vu que c'est un outil qui va permettre de faire des croisements, je suppose, au niveau du ministère, notamment avec les lettres de mission, on s'est dit que finalement, 1607 heures pour nous, qui est le quota annuel, on va prouver que c'est très très vague. Nous, on fonctionne à la mission, on ne regarde pas le temps qu'on passe à une mission. On est susceptible de partir pendant une semaine en compétition, même deux semaines de suite, donc, qu'est-ce qui se passe au niveau de la récup ? Donc, on met tout cela sur l'agenda qu'on remplit et puis ça peut faire évoluer peut-être les esprits des gens qui vont regarder, qui sont peut-être un petit peu moins sur le terrain avec nous et qui ne se doutent pas de la charge de travail qu'on peut avoir à un certain moment. » (Un CTS, 5 ans d'ancienneté)

Cet instrument est d'autant plus mal perçu par les CTS qu'il ajouterait aux tentatives déjà récurrentes d'instauration de rapports "serviciels» dont ils sont l'objet de la part des dirigeants sportifs. Le fait que ces professionnels ne soient ni détachés ni mis à disposition des fédérations,

${ }^{51}$ QUILLIEN, Claudie. 2012. Rapport de la mission d'étude relative à la constitution du vivier des emplois de directions et à l'accompagnement des carrières. Rapport $\mathrm{n}^{\circ}$ 2012-M-04, Inspection générale de la jeunesse et des sports, p. 7.

${ }^{52}$ Le SNAPS considère que «l'outil n'est en l'état pas adapté à l'exercice de nos missions ; sa conception relève d'un «idéal rationalisateur ». Or les spécificités techniques et culturelles de chaque fédération, sa taille et l'effectif des CTS rend toute visée standardisante totalement illusoire », in SNAPS Infos, $\mathrm{n}^{\circ}$ 96, octobre 2013, p. 19. 
mais "placés auprès » d'elles, a été justifié par la volonté de sécuriser les missions de ces cadres chargés de concevoir, proposer aux dirigeants fédéraux et mettre en œuvre des politiques sportives qui imposent de la continuité pour être performantes : sport de haut niveau, formation des cadres et développement de toutes les formes de pratiques sportives ${ }^{53}$. À ce sujet, si un représentant syndical rappelle que le principe de mise à disposition a été écarté, car «il aurait donné un droit de vie ou de mort des CTS au mouvement sportif», ce particularisme n'exclut pas pour autant les stratégies de contrôle du travail des CTS de la part des dirigeants fédéraux voire des possibilités de relégation de ces personnels. En ce sens, les périodes d'instabilité que connaissent les DTN, notamment lors du renouvellement des présidents de fédérations, sont régulièrement évoquées pour les illustrer ${ }^{54}$. Parce qu'elles concernent toutes les catégories de CTS, les représentants des organisations syndicales admettent que l'administration centrale peine à gérer les situations de conflits que cela engendre avec les dirigeants sportifs. Ils indiquent par ailleurs qu'elles se soldent par une réaffectation de missions le plus souvent subie par les CTS, ce qui rend leur parcours professionnel très incertain ${ }^{55}$.

Si la figure du PTP qui se dégage de ces éléments se caractérise par une réduction de ses marges d'autonomie, ils donnent également à voir une dissolution des collectifs de travail.

\section{b) Une individualisation des tâches et une segmentation du groupe renforcées}

Les injonctions plus ou moins fortes à exercer différemment leur activité professionnelle ou à en rendre compte, parce qu'elles percutent les professionnalités établies, font régulièrement l'objet de réactions syndicales. La revendication de l'autonomie d'action des PTP étant placée au

\footnotetext{
53 Bouchout J.-P. ; Jarrige B. ; Primault J. ; Watrin D., op. cit.

${ }^{54}$ Quillien C., op. cit., p. 7.

${ }^{55}$ HontA, Marina ; JulHe, Samuel, 2013. «Les professions du secteur public saisies par la privatisation. Le cas des Conseillers techniques sportifs », Gouvernement et action publique, $\mathrm{n}^{\circ} 1$, p. 63-87.
} 
cœur du débat ${ }^{56}$, ces porte-parole du groupe n'hésitent pas à se mobiliser (bulletins syndicaux informant les agents de leurs droits, connaissance très pointue du cadre réglementaire les précisant, rappel systématique de ces droits auprès de représentants de l'administration), dès lors qu'ils estiment que certains chefs de service « dérapent ${ }^{57} »$. Ces formes constantes de mobilisation corporatistes ne sauraient masquer que ce groupe professionnel présente, lui aussi, des contours flous et un important degré de segmentation ${ }^{58}$ que les changements induits par les réformes administratives accentuent. Plusieurs facteurs témoignent ainsi des « sous-cultures » qui le composent alors même que la définition du statut, acquis sur titre par concours, semble n'autoriser qu'une seule forme identitaire.

Depuis la constitution du corps des Professeurs de sport, en 1985, la position administrative et fonctionnelle des CTS a plusieurs fois fait l'objet d'aménagements réglementaires, ces dispositions leur octroyant des marques de distinction. Les conditions d'accès à la profession en premier lieu sont pour partie distinctes dans la mesure où les épreuves d'admission diffèrent en fonction de la spécialité choisie (CAS ou CTS). Le " placement des CTS auprès » des fédérations, régime dérogatoire au droit de la fonction publique que la Cour des comptes qualifie par ailleurs de "positionnement imprécis ${ }^{59}$ ", a ensuite été institué pour apporter un renforcement des compétences techniques dans ces organisations sportives et conforter ainsi leurs actions tant aux plans national qu'international. Au regard de ces éléments, il faut se garder de trop forcer le trait coercitif que les dirigeants sportifs font peser sur leur activité dans la mesure où, pour quelques CTS, le placement sous le contrôle de la puis-

\footnotetext{
56 Paradeise, Catherine. 2008. «Autonomie et régulation : retour sur deux notions clés », in LE BIANIC T., VION A. (dir.), op. cit., p. 289-296.

${ }^{57}$ Lire par exemple, LERNOULD, Claude. 2013. «L'autonomie ou la mort... », SNAPS Infos, n 94, p. 13-14; KRUMBHOLZ, Jean-Paul. 2014. «L'avenir de J\&S au sein des DDI plus que jamais en débat. Pendant que le nouveau gouvernement réfléchit, certains directeurs départementaux dérapent », SNAPS Infos, n 99, p. 6.

${ }^{58}$ BuCHer, Rue ; STRAuss, Anselm. 1961. "Professions in process », American journal of sociology, $\mathrm{n}^{\circ}$ 66-4, p. 325-334.

${ }^{59}$ COUR DES COMPTES. 2013. Sport pour tous et sport de haut niveau : pour une réorientation de l'action de l'État. Rapport thématique, p. 135.
} 
sance fédérale est vécu comme une évidence, voire une préférence. Leur parcours de pratiquant sportif et celui de leur formation initiale ${ }^{60}$ font que ces agents sont directement issus de ce secteur et se sentent parfois redevables d'une large part de leur carrière, ces instances (clubs, comités sportifs départemental ou régional) leur ayant fréquemment offert leur premier emploi. Par ailleurs, des CTS perçoivent des régimes de rémunération complémentaires qui les placent eux aussi sous l'influence, voire sous la dépendance des responsables fédéraux et les exposent, par ailleurs, à des conflits d'intérêts. Faisant là encore et régulièrement part de son étonnement à l'égard de telles pratiques que les dirigeants justifient par la volonté de conserver des cadres de valeur dans un environnement international concurrentiel, la Cour des comptes souligne néanmoins que ces compléments soulèvent des interrogations dans la mesure où les intéressés cumulent pour les mêmes fonctions une rémunération publique et une rémunération privée sans que cette dernière soit encadrée. Aussi, quel que soit son montant, le complément de rémunération opère inévitablement une remise en cause de la neutralité statutaire des PTP fonctionnaires puisqu'il les place de fait sous la subordination directe des fédérations ${ }^{61}$.

Dans ce contexte, et par lettre ministérielle datée du 23 mai 2014, l'Inspection générale de la jeunesse et des sports (IGJS) a été sollicitée pour mettre en place une mission d'appui auprès de la Direction des sports en vue d'élaborer un projet de code de déontologie des PTP exerçant les missions de CTS. Si l'enjeu est ici de prévenir les risques professionnels engendrés par ces relations complexes avec les élus sportifs, le document de travail précise également les obligations qui s'imposent à eux (règles générales de cumul, respect de la hiérarchie, devoir de loyauté, etc.). Il rappelle aussi les principes de base selon lesquels les agents publics doivent être exemplaires dans l'exercice de leurs fonctions et

\footnotetext{
${ }^{60}$ L'obligation qui leur a été faite d'être titulaire d'un Brevet d'État d'Éducateur Sportif du second degré (décret n79-474 du 7 juin 1979 fixant les dispositions applicables aux conseillers techniques et pédagogiques de la jeunesse, des sports et des loisirs), dont les contenus d'enseignement sont en grande partie définis par le mouvement sportif, caractérise leur trajectoire de formation initiale.
}

${ }^{61}$ Ibid., p. 137. 
porteurs de valeurs ou obligations de neutralité, d'impartialité, de probité, de réserve et de laïcité.

Outre ces aspects, la division du travail à la fois verticale (entre CTPS et Professeurs de sport) et horizontale ${ }^{62}$ participe aussi pleinement de cette segmentation. Une telle spécialisation fonctionnelle fait que l'identité professionnelle des CTS a toujours été plus facile à valoriser à travers le crédit réputationnel procuré par la médiatisation des résultats des athlètes ou équipes qu'ils encadrent dans les compétitions internationales. Aussi, parce que cette fraction de PTP passe pour celle qui exerce la mission la plus noble, c'est en tant que «vitrine » du ministère des Sports que les représentants du SNAPS interrogés la présentent. À l'inverse, les fonctions de CAS sont plutôt dévalorisées voire stigmatisées par les CTS qui les perçoivent généralement comme une voie possible de mobilité professionnelle pour leur fin de carrière, "quand ils seront usés ». Les réformes administratives ont contribué à renforcer cette « hiérarchisation morale du travail ${ }^{63} »$. Que les PTP en aient fait l'expérience ou pas, les tâches en DDCS/DDCSPP sont perçues et/ou vécues comme étant les moins valorisantes en raison des tensions qui existent entre leur caractère pléthorique et les ressources toujours plus limitées à disposition. Avec la réduction des effectifs notamment, les moyens mobilisables pour mettre en œuvre les politiques ministérielles sont faibles alors que le périmètre des missions fixées par les directives nationales d'orientations (DNO) qui incombe au réseau DRJSCS/DDCS ou DDCSPP est, lui, resté le même. Une telle diversité de tâches, bien plus réduite en direction régionale en raison d'équipes plus étoffées, met à mal les capacités des PTP à les exercer ${ }^{64}$ :

\footnotetext{
${ }^{62}$ Entre spécialités (formation, développement des pratiques, préparation à l'excellence sportive) et lieu de leur exercice (services déconcentrés, CREPS, fédérations, ligues ou comités régionaux).

${ }^{63}$ Hugues E. C., op. cit.; BIDET Alexandra. 2011. L'engagement dans le travail. Qu'est-ce que le vrai boulot?, Paris, PUF.

${ }^{64}$ Ces aspects confirment une situation objectivée par la Cour des comptes en 2013 : la poursuite de la baisse des effectifs en 2013 expose en effet les services à de très fortes inégalités quant à leurs possibilités d'intervention : les effectifs réels de CAS, bien qu'au nombre de quatre en moyenne dans chaque DDCS/DDCSPP, ne coïncident pas avec les effectifs indicatifs. Dans ce contexte et concernant la DDCSPP de l'Orne par
} 
«On a fusionné deux administrations qui ne travaillent pas du tout de la même façon, car la DDASS, c'est essentiellement une logique de traitement de dossiers en bureau or, nous, ça n'est pas que cette logique! On doit avoir une logique de réaction par rapport au terrain. Mais sur le CNDS on a également cette logique maintenant, car on n'est plus que trois à la $\mathrm{DD}$. On ne va plus voir les clubs pour les aider et on ne les reçoit plus, car il n'y a plus le temps, on fait juste une information auprès des comités. On voit bien qu'on en vient à cette logique de traitement: "il y a un appel à subvention, vous envoyez les dossiers, on instruit les dossiers et on vous dit combien à la fin.” » (Un CAS en DDCS, 9 ans d'ancienneté)

Ce manque de moyens déstabilise la constitution ou la préservation de collectifs forts de travail. L'individualisation des tâches qui en découle n'étant que peu propice à la coopération sociale, c'est avant tout à travers des stratégies individuelles que les PTP en charge de plusieurs dossiers cherchent à préserver leur autonomie en essayant de moduler leur investissement professionnel en fonction de leur intérêt et goût pour telle ou telle mission. Dans ce contexte, l'un des CAS rencontrés, venant d'obtenir une mutation en DR et professeur de sport depuis cinq ans, revient sur ses conditions de travail en DD et sur ses interrogations quant au sens à donner à ses fonctions :

«C'était devenu super compliqué de par la diversité des missions qu'on te donne. J'étais référent de 6 dossiers l'an passé, ce qui est juste pas possible. Nos DNO, c'est un millefeuille d'actions! Le matin, tu as un appel sur le CNDS; l'après-midi, tu as une réunion sur sport et handicap et le soir tu vas sur les réglementations sportives ou des choses comme ça. Donc après, t'essayes dans ta tête de trouver du lien. J'ai essayé de relier les missions les unes aux autres parce que si tu les déconnectes, ça devient très compliqué. Malheureusement, du fait des effectifs dans les services, à un moment donné, tu

exemple, seuls sept contrôles d'établissements avec un seul agent ont été menés sur une base de 915 établissements déclarés. Le contrôle des équipements est encore plus réduit. 
es plus sur l'urgence donc tu n'as pas le temps de te projeter. Ça, pour moi en tout cas, ça a été horrible ».

Parce qu'ils se sentent moins disponibles pour leurs partenaires, les PTP craignent de produire eux aussi des services «bas de gamme ${ }^{65}$ » et ce faisant, que leurs difficultés soient pointées comme des problèmes de performance dont ils seraient seuls responsables. Ce manque de moyens participe ainsi et plus généralement de l'affaiblissement de la légitimité professionnelle des PTP alors que les transformations de l'action publique les amènent à devoir composer avec une multiplication d'acteurs se mobilisant dans le domaine sportif.

\section{III- Des missions mises à mal par une division du travail marquée}

Bien que les PTP ne soient pas affectés de la même façon par les processus à l'œuvre, cet espace désormais complexe et concurrentiel qu'est l'organisation des APS les expose tous à la difficulté de valoriser des niches de compétences qui seraient insubstituables. Plusieurs d'entre eux estiment par ailleurs que les transformations de l'action publique n'ont pas encore produit tous leurs effets. En ce sens, l'accentuation du processus décentralisateur déjà fortement problématique pour cette administration et les revendications du mouvement sportif quant à la gestion des carrières des CTS, font peser, ici aussi, des doutes sur la définition des missions que ces agents auront à assumer et sur les modalités organisationnelles de leur exercice ${ }^{66}$. Dans ce contexte, la politique de «redéploiements » récemment amorcée pour les CTS consacre, dans ce secteur également, une évolution des métiers sources (les missions techniques et pédagogiques) vers les métiers cibles (celui du «manager ») et entérine, par là même, le déclin de la suprématie technique de l'ensemble du groupe des PTP $^{67}$.

\footnotetext{
${ }^{65}$ BeLORGEY N., op. cit., p. 33.

66 SCHWEYER François-Xavier; CAMPEON Arnaud. 2008. «Le pouvoir de régulation à l'épreuve de la division du travail : les médecins inspecteurs de santé publique », in LE BIANIC T. ; VION A. (dir.), op. cit., p. 73-89.

${ }^{67}$ Dumoulin Céline. 2010. « Puissance et déclin des techniciens à France Télécom », in Boussard V. ; Demazière D. ; Milburn P., op. cit., p. 35.
} 
Sciences sociales et sport, $n^{\circ}$ 9, 2016, p. 115-152

\section{a) La décentralisation ou la crainte du renforcement de la mise en péril des missions}

La RéATE a amené les préfets de département et/ou les chefs de service à progressivement prioriser l'intervention des DDCS/DDCSPP sur quelques dispositifs particuliers ${ }^{68}$ comme la gestion des crédits du CNDS ou des programmes d'aides à l'emploi, mais aussi sur des missions d'inspection et de contrôle liées à la sécurité des APS (contrôle des établissements, des manifestations sportives, de l'exercice de la profession d'éducateur sportif), axes présentés comme étant les principaux, voire les seuls centres d'intérêt de la hiérarchie dans ce domaine. Cette polarisation, que les PTP disent par ailleurs comprendre en raison, là encore, de la faiblesse des effectifs, se fait au détriment des missions d'accompagnement et d'expertise :

«Pour avoir connu quelques inspecteurs, ils sont bien dans le moule de la répression aujourd'hui. Les centres de loisirs et établissements d'APS, on n'y va que pour contrôler, pas du tout pour conseiller. Même à un moment donné, dans une réunion de service, ils m’ont dit : "Oui, le contrôle des centres de loisirs, ça fait partie de... c'est au cœur de vos missions." Je dis : "Non, peut-être les vôtres, mais pas les miennes." C'est au cour des missions des DDI maintenant, effectivement, peut-être, mais pas du corps des professeurs de sport. Quand tu as des gens qui sont comme ça, là tu peux difficilement avancer. » (Un CAS en DDCSPP, cinq ans d'ancienneté)

Face à ces situations qui dévalorisent et déstabilisent la portée de ce qu'ils faisaient jusqu'à présent, ils estiment que leurs compétences sont désormais sous-exploitées. Les missions priorisées par leur hiérarchie seraient ainsi en décalage non seulement avec leur qualification, mais aussi avec le contenu des politiques sportives du ministère. Dès lors, la dégradation exprimée de leurs conditions de travail provient avant tout du fait qu'ils peinent à accepter l'idée que «l'action de terrain », ou plus précisément leurs missions techniques et pédagogiques soient désormais menacées, car perçues comme accessoires. Ainsi, et au même titre que les

68 COUR DES COMPTES. 2013. L'organisation territoriale de l'État, rapport public thématique. 
personnels des anciennes Directions départementales de l'équipement ${ }^{69}$, ils craignent que ce ministère et les activités qui constituaient leur identité professionnelle ne disparaissent. Ce sentiment de déclin, associé ici à l'affaiblissement, voire à la perte des missions qui ont forgé leur reconnaissance et crédibilité, est accentué par le fait que dans plusieurs services, certains dossiers ne sont plus portés que par des contractuels. L'absence de perspectives de renouvellement de ceux-ci accroit le malaise dans la mesure où elle engendre la perception d'une incapacité à les assumer suite à leur départ.

Ces injonctions normatives entamant la maîtrise de l'activité des PTP tout autant qu'elles réduisent là encore leur autonomie, ajoutées aux cultures professionnelles diverses, font qu'aujourd'hui, ils ne perçoivent aucune unité dans les services au sein desquels ils exercent leurs fonctions. De ce fait, l'accentuation du travail interministériel que devait produire la réforme demeure-t-elle encore entièrement à construire :

«Travailler dans l'interministérialité, à mon avis c'est encore un leurre. Mais après, c'est vraiment la volonté des agents de vouloir travailler ensemble et elle se développe grâce à des moments conviviaux et des moments de connaissance des missions de chacun. Pour l'instant, on n'y est pas vraiment encore... Par exemple, dans mon ancien service, c'était vraiment flagrant parce que tu avais le couloir et une porte au milieu. Tu avais d'un côté tous les ex-DDASS et de l'autre, tous les ex-jeunesse et sport. Et il n'y avait aucun lien. Je pense que pour beaucoup, c'est par méconnaissance des missions et de l'intérêt qu'il peut y avoir à faire des choses en commun. Des tensions qu'il peut y avoir aussi de par les différences de statuts. » (Un CAS en DRJSCS, trois ans d'ancienneté)

Devant ces constats et pour mettre en œuvre une politique ministérielle qualifiée d'ambitieuse en matière sportive, les organisations syndicales et les rapporteurs de missions d'inspection, revendiquent désormais de privilégier les «compétences métiers » des corps propres en relançant une politique de recrutement volontariste par l'organisation régulière des

\footnotetext{
${ }^{69}$ BertauX-Wiame Isabelle ; DiverT Nicolas. 2006. «La direction départementale de l'équipement. Étude de cas », in LINHART D., op. cit., p. 71-130.
} 
concours avec un nombre significatif de places et en renforçant également les prérogatives du niveau régional en matière de pilotage de l'organisation territoriale de l'État. Sur ce dernier point, et partant du principe que les PTP, dans les DDCS/DDCSPP où ils n'ont pas trouvé leur place, "souffrent» chaque jour davantage ${ }^{70}$, le SNAPS demande leur affectation en DRJSCS, affectation qui, selon eux, serait la mieux à même de permettre le maintien des missions de proximité. La proposition peut revêtir un caractère paradoxal dans la mesure où géographiquement elle peut représenter un éloignement, notamment dans des régions très vastes ou marquées par la présence d'obstacles physiques (reliefs, vallées). En outre, pour les acteurs locaux du sport, l'interlocuteur régional n'est pas toujours aussi clairement identifié que les services départementaux. Les représentants de l'Inspection générale proposent, quant à eux, le maintien d'une présence institutionnelle dans les départements, mais à travers la création d'unités territoriales (UT) comme l'ont fait d'autres ministères. Il s'agit ici de "garantir une expertise territorialisée fine $^{71} »$ et, ce faisant, de restaurer, à cette échelle, la visibilité des missions des PTP et plus généralement de cette administration.

Ces débats restent encore ouverts, car d'autres facteurs que les PTP perçoivent comme menaçant l'avenir de leur institution confortent cette situation d'incertitude. De nombreux agents en poste dans les services déconcentrés, les établissements et les CREPS craignent en effet les changements qui pourraient découler de l'actuelle réforme territoriale. Le transfert possible aux collectivités territoriales, notamment aux Régions, de responsabilités en matière de formation professionnelle, transfert justifié par le fait qu'il favoriserait une rationalisation dans l'exercice de ces prérogatives, puisque ces collectivités sont déjà largement compétentes en la matière, est perçu comme une orientation qui influerait immanquablement sur les missions des agents mobilisés dans le champ des formations aux métiers de l'animation et de l'entraînement sportif.

Par ailleurs, des représentants de l'Inspection générale reconnaissent que le ministère n'est plus en mesure d'assumer seul l'entretien, la réno-

\footnotetext{
${ }^{70}$ JEAN Caroline. 2014. «Bilan du Conseil national de Nancy», SNAPS Infos, n 97 , février, p. 15.

${ }^{71}$ Bouchout J.-P. ; Jarrige B. ; Primault J. ; Watrin D., op. cit., p. 118.
} 
vation, voire le remplacement de l'immobilier de ses $17 \mathrm{CREPS}^{72}$. Les besoins sont en effet très importants tant en matière de modernisation et d'adaptation des conditions d'hébergement des sportifs accueillis dans les pôles France et Espoir, que de rénovation et/ou reconstruction des équipements sportifs et d'accompagnement de la performance (plateau médical, récupération, musculation spécifique). Face à ce constat, une nouvelle réforme du réseau des CREPS est engagée ${ }^{73}$. Présentée comme visant à «renforcer leur ancrage territorial autour d'un projet partagé avec les collectivités locales ${ }^{74} »$, elle [les transforme] en établissements publics locaux de formation du sport, de la jeunesse et de l'éducation populaire, et en confie la gestion administrative et immobilière aux conseils régionaux sur le modèle partiellement inspiré des établissements publics locaux d'enseignement (EPLE). Si ce dernier point est de nature à rassurer les agents exerçant leurs fonctions dans ces établissements, il reste que les craintes d'ingérence des Régions dans la définition de l'action des CREPS sont évoquées dans la mesure où elles voient en effet leur représentation renforcée au sein du conseil d'administration. Ces craintes renvoient aussi au fait que les PTP admettent, tout autant qu'ils le regrettent, que la mise en œuvre de l'action sportive ministérielle repose déjà fortement sur la participation des collectivités territoriales. De telles perspectives pourraient encore accentuer cette dépendance et, ce faisant, la fragilité de l'institution ministérielle :

«Nos contraintes, c'est plus en termes de relations, notamment avec la ville de L. sur la gestion des équipements où on est fortement dépendants d'eux parce qu'on n'a pas d'équipement sportif, nous, directement, pour pouvoir proposer les temps de formation, la mise en place des séances. On est dépendants de la ville. Donc, quand ça se passe bien, ça va, quand c'est un peu plus tendu, c'est plus difficile. Si du jour au lendemain, le maire dit: "On ne vous met plus à disposition les piscines et on ne vous met plus à disposition les équipe-

\footnotetext{
72 Ibid.

${ }^{73}$ Loi no 2015-991 du 7 août 2015 portant nouvelle organisation territoriale de la République.

74 Actus JS, La lettre des services et agents du ministère des Sports, de la Jeunesse, de l’Éducation populaire et de la Vie associative, Spéciale MAP, juillet 2013, p. 4.
} 
ments sportifs pour la formation", on met la clé sous la porte. On est obligés d'aller voir sur une autre collectivité ce qui veut dire qu'après derrière, c'est les stagiaires qui doivent se déplacer et ainsi de suite. Donc c'est une des contraintes qui est toujours présente suivant les relations que vont avoir la collectivité et la direction régionale. » (Un CAS en DRJSCS, cinq ans d'ancienneté)

Ces réactions démontrent aussi qu'en l'absence de répartition de compétences clairement définie, la question de la coexistence entre deux filières territoriales de gouvernement du sport continue d'interroger. Dans ce contexte, les PTP estiment que l'action de l'État devrait être redéfinie afin qu'elle s'affranchisse des logiques d'intervention des collectivités qu'ils n'hésitent pas à disqualifier. Plus généralement, s'ils estiment légitime que "chacun veuille faire sa place », ils regrettent toutefois que cela soit souvent «contre-productif», car « on est sur des champs tellement partagés ». Ici aussi, ces agents, bien qu'attachés à leur métier, sont également ouverts à des réaménagements de leurs missions ${ }^{75}$. L'un d'eux indique en ce sens :

« Il faudrait que l'on clarifie un petit peu les missions de l'État. Qu'on ne fasse pas tous la même chose. À mon sens, peut-être que l'État doit être sur la définition de l'élément de cadrage ou de contrôle ; faire en sorte qu'il soit en capacité de définir des conditions de réalisation d'un projet et laisser faire les acteurs de proximité. Bien sûr, ils sont légitimes pleinement pour le faire, mais qu'ils le fassent beaucoup mieux... On a besoin de clarification, de simplification: qui fait quoi ? On aurait besoin, me semble-t-il, d'un petit peu d'équité, de neutralité, d'un arbitrage, un rôle qui devrait revenir à l'État. » (Un CAS en DDCS, 15 ans d'ancienneté)

Ces projections sur un État neutre et régulateur dont ces agents seraient les garants de par leur identité de fonctionnaire, constituée là aussi à distance des intérêts particuliers, sont présentes chez toutes les catégories de PTP interrogés. Elles sont en effet tout aussi fortes dans les récits des CTS rencontrés.

${ }^{75}$ LINHART D., op. cit. 


\section{b) Une « fin de règne » douloureusement vécue}

Alors que les effectifs de CTS présentent la particularité d'avoir très peu varié durant les 20 dernières années, les concernant, ce sont surtout les reconfigurations des relations entre l'État et le mouvement sportif qui questionnent la redéfinition de leurs missions, les modalités de leur affectation et de leur gestion de carrière.

La dynamique, bien qu'inégale, de croissance des ressources commerciales des fédérations et les programmes publics d'aides à l'emploi ont permis le recrutement, en leur sein, d'entrâneurs salariés. La coexistence entre ces derniers et les CTS, si elle est précieuse pour traiter des diverses missions induites par la division du travail au sein des organisations sportives associatives, accroît les difficultés de positionnement des PTP vis-àvis des dirigeants sportifs, des autres salariés et des athlètes encadrés. Elle déstabilise par là même leur « dominance structurelle ${ }^{76}$ ", ce concept désignant un état de la relation entre professionnels - ou groupes professionnels - qui coopèrent pour l'accomplissement d'une tâche sociale tout en étant en concurrence pour le contrôle de cette tâche. Ceci contribue à rendre incertains les attributions et rôles de ces deux catégories de travailleurs au sein d'une même organisation fédérale et invite à revoir les territoires, autrefois monopolistiques, d'intervention des CTS ${ }^{77}$. Autrement dit et alors qu'ils étaient avant tout des «leaders» reconnus pour leurs compétences techniques - mission qui forgeait et forge encore non seulement leur "passion au travail », mais aussi leur réputation -, les CTS peuvent effectuer désormais des tâches très différentes, tâches variant fortement d'une fédération à l'autre et présentant, pour eux, un intérêt très variable là encore ${ }^{78}$. L'idée, en outre, que ces salariés de droit privé puissent à terme les remplacer dans l'exercice de leurs missions les amène, eux aussi, à insister sur ce qui fait leur spécificité :

\footnotetext{
${ }^{76}$ FREIDSON Eliot. 1970. Professional dominance: the social structure of medical care, New York, Aldine Publishing Company.

77 AвBOTT Andrew. 2003. "Écologies liées : à propos du système des professions ", in MENGER Pierre-Michel (dir.). Les professions et leurs sociologies. Modèles théoriques, catégorisations, évolutions, Paris, MSH, p. 29-50.

${ }^{78}$ HontA M. ; Julhe S., op. cit.
} 
leur identité de fonctionnaire d'État garant de valeurs éthiques particulièrement menacées dans le secteur de l'excellence sportive ${ }^{79}$ :

«Est-ce que le corps va s'éteindre ou pas de sa belle mort progressivement? Le monde dans lequel ce corps a été créé, il a bien évidemment changé. 1960, ce n'est pas 2020, ce n'est pas 2012, il y a de l'eau qui a coulé sous les ponts. Par contre, il serait quand même intéressant, avant de penser à ne faire qu'une analyse financière du dispositif, de faire une analyse globale et complète des effets obtenus dans tous les domaines : de la formation, du sport de haut niveau, du développement des fédérations, surtout sur la question du sport facteur d'intégration sociale, de la vie associative et du sport en général, en liaison avec la santé... Quand cette analyse sera faite, on pourra peut-être voir ce qui a changé et ce qu'on peut faire. Je suis très sincèrement convaincu que nous restons les derniers garants d'une forme de recul et de neutralité nécessaires par rapport aux enjeux engagés dans ces missions-là : des missions de responsabilité et de pouvoir qui font que cela peut monter à la tête, qu'il n'est jamais bon de mettre tous les œufs dans le même panier, et qu'on va déjà suffisamment vers l'économie de marché pour s'arrêter là. » (Un CTS, 22 ans d'ancienneté)

Dans ce contexte, les dirigeants fédéraux, sans remettre en cause l'existence des CTS, condamnent néanmoins le fait que le ministère des Sports les utilise pour orienter les politiques fédérales et contrecarrer, parfois, des initiatives d'élus sportifs. Ce qui est ici contesté est le «lobby» qu'ils constitueraient soit pour manifester une solidarité sans faille de nature corporative vis-à-vis des leurs, soit pour freiner les orientations souhaitées par une partie des élus fédéraux ${ }^{80}$. Dès lors, des dirigeants comme le président de la Fédération française d'athlétisme attendent du ministère qu'il leur permette de gérer directement la carrière des CTS

\footnotetext{
${ }^{79}$ Afin de conforter cette identité et de restaurer ce faisant la visibilité sociale des CTS, le SNAPS « revendique l'utilisation du vocable «Cadre technique d'État» (CTE), sans spécification régionale ou nationale », SNAPS Infos, $\mathrm{n}^{\circ}$ 97, p. 18.

${ }^{80}$ Lavaure Patrick; Petrequin Luc; Watrin Daniel. 2013. Mission relative au partenariat entre l'État et le Comité national olympique et sportif français (CNOSF), rapport 2013-M11, Paris, Inspection générale de la jeunesse et des sports.
} 
afin que le cadrage de leur activité professionnelle soit exclusivement soumis à leur approbation ${ }^{81}$. Si ce dernier, relayé par les organisations syndicales et les conclusions de diverses missions d'inspection, refuse de céder à ces revendications externes, elles sont largement connues des CTS. En ce sens, leurs craintes quant à une possible modification de leur statut sont réelles :

«C'est assez flou, je trouve... Moi, j'ai l'impression qu'à un moment donné, on va devoir faire un choix entre le service public ou le privé, la fédération. Moi, je le sens. Je ne sais pas si je me trompe ou quoi, mais j'ai l'impression que cela peut arriver. Et je ne sais pas trop ce que je ferai. » (Un CTS, 11 ans d'ancienneté).

Enfin, et suite notamment aux demandes répétées de la Cour des comptes $^{82}$ pour que soit amélioré le suivi de ce dispositif, un redéploiement des CTS a progressivement été adopté. Afin d'instaurer une répartition "plus équitable », il vise à les affecter prioritairement auprès des fédérations ne disposant pas ou pas suffisamment de ressources pour recruter leurs propres salariés. Ce processus, consistant à placer auprès des fédérations des CTS pouvant être des non-spécialistes de la discipline sportive en question, s'est ainsi accentué ces dernières années. Il est justifié par le fait qu'il n'est plus attendu d'eux qu'ils restent au cœur de la production de la performance sportive, mais qu'ils endossent désormais le rôle de managers chargés de la stratégie technique de long terme ${ }^{83}$, rôle mobilisant d'autres compétences que celles strictement techniques. Être placé auprès de ces organisations sans pour autant être un spécialiste de la discipline suscite des réserves de la part des CTS, un tel processus pouvant accroître encore leurs difficultés de positionnement à l'égard des dirigeants sportifs et des autres catégories de salariés au sein des fédérations. Cela implique que le CTS ait à justifier là aussi de ses compétences $^{84}$, préalable indispensable à la (re)construction de la reconnais-

\footnotetext{
${ }^{81}$ COUR DES COMPTES. 2009. op. cit.

82 Ibid.

83 Bouchout J.-P. ; Jarrige B. ; Primault J. ; Watrin D., op. cit.

${ }^{84}$ De LuCA Virginie. 2001. "Justifier ses compétences : un réflexe de défense professionnelle des inspecteurs des enfants assistés à la fin du XIX ${ }^{\mathrm{e}}$ siècle », Sociologie $d u$ travail, $\mathrm{n}^{\circ} 43$, p. 111-129.
} 
sance de son travail surtout dans un milieu où se sont forgées des réputations différenciées ${ }^{85}$ :

«Les gens qui ont beaucoup d'expérience, qui sont sur des postes importants, ils peuvent repartir sur d'autres fédérations. Parce qu'ils ont l'expérience, ils ont un vécu, donc ils sont connus. Après, les gens qui ont un peu moins d'expérience, des gens comme moi, par exemple, je ne sais pas comment je pourrais faire pour me recycler dans une autre fédé. Il faudrait que j’aie une valeur ajoutée importante sur un domaine particulier. Cela me plairait bien moi, je ne le mets pas de côté pour la suite, mais il faudrait que j'aie quelque chose qui puisse vraiment aider la fédé concernée. »(Un CTS, six ans d'ancienneté)

Tous ces éléments expliquent que la révision des modes de gestion de la carrière des CTS est régulièrement préconisée pour réduire les diverses conséquences de l'ambiguïté organisationnelle dans laquelle ils se trouvent. Aussi et afin de construire les conditions favorables à la transmission interdisciplinaire rendue nécessaire par le processus de redéploiements des CTS et éviter que s'accroissent plus encore les tensions imputables à ces situations de travail complexes ${ }^{86}$, la Direction des sports du ministère a créé en 2013 un « centre de gestion opérationnelle des cadres techniques sportifs »(CGO-CTS). Service à compétence nationale, il a pour mission d'assurer la gestion prévisionnelle des emplois et des CTS, de suivre la répartition de leurs effectifs sur le territoire national et entre les fédérations et de proposer, en lien avec l'INSEP, les orientations et les objectifs de leur formation initiale et continue. Si la responsable de ce service le présente comme «un véritable service de proximité pour les agents auxquels il doit offrir une gestion plus personnalisée de leurs situations ${ }^{87}$ ", les principaux intéressés ne perçoivent pas encore, en raison de sa récente création, les enjeux et l'intérêt de son existence.

\footnotetext{
${ }^{85}$ KARPIK Lucien. 2007. L'économie des singularités, Paris, Gallimard.

${ }^{86}$ Bouchout J.-P. ; Jarrige B. ; Primault J. ; WATrin D., op. cit. ; LAVAure Patrick ; WATRIN Daniel. 2011. Rapport relatif aux missions des cadres techniques sportifs placés auprès des fédérations sportives, Paris, Inspection générale de la jeunesse et des sports.

${ }^{87} \mathrm{La}$ directrice de ce service ajoutant : «Les CTS sont une ressource essentielle pour le sport français et notre objectif est de préserver ce capital humain, de les accompagner,
} 
Quand le mandat s'étiole

\section{Conclusion}

Bien qu'il soit encore possible d'identifier le ministère des Sports comme une institution dont les agents possèdent une identité de métier, les PTP sont très préoccupés par le risque d'une disparition de leur administration et redoutent les changements à venir. Parmi ceux-ci, l'attribution de compétences supplémentaires aux collectivités territoriales (formation professionnelle, gestion des CREPS), dont le contenu reste encore très imprécis, et le durcissement des tensions entre le ministère des Sports et le mouvement sportif leur font émettre de sérieuses réserves quant à la qualité et la neutralité de ce que pourrait être demain le service public du sport. Alors que leurs activités techniques et pédagogiques leur donnaient une crédibilité professionnelle positive et forte, les PTP ont aujourd'hui parfaitement conscience que d'autres catégories de professionnels disposent de ces mêmes compétences (les cadres de la filière sportive de la fonction publique territoriale pour les CAS, les salariés recrutés sous contrat de droit privé par les fédérations et leurs organisations territoriales pour les CTS), ce qui renforce leur sentiment de perte de visibilité sociale. S'ils ont tous compris l'enjeu que représentait le fait de pérenniser, à défaut de maitriser, leurs relations au mouvement sportif, les injonctions à demeurer dans les services pour les CAS et le déclin du «monopole de juridiction » des CTS, les empêchent de toujours mener à bien ce projet. Dans ce contexte, leurs réflexes corporatistes visant la préservation des acquis, relayés par ailleurs par les organisations syndicales, parce qu'ils obèrent leurs capacités collectives à renouveler les formes de leur reconnaissance et légitimité professionnelles, rendent difficile la définition d'une mission qui serait reconnue comme indispensable. Ainsi, et au-delà de l'étiolement du mandat, les transformations de l'action publique peuvent, dans la mesure où elles conduisent directement à raisonner en termes de métiers et de compétences et moins en termes de rattachement institutionnel, provoquer également l'érosion de leur statut ${ }^{88}$.

les défendre au besoin et d'être au plus près d'eux lorsqu'ils en ont le plus besoin, et de favoriser la préservation et le développement de leur expertise. »

88 SCHWEYER F.-X; CAMPEON A., op. cit., p. 76. 
Bien que les PTP peinent à accepter ces glissements qui effritent la confiance et la reconnaissance des partenaires associatifs, ils les estiment aussi irréversibles. Pour autant, les stratégies d'exit vers les collectivités territoriales ou le secteur privé massivement observées dans d'autres administrations ${ }^{89}$ sont peu envisagées ici, même si les cas de CTS acceptant d'entraîner, en France ou à l'étranger, des équipes de clubs ou des athlètes existent depuis plusieurs années. De telles stratégies contribuent pleinement elles aussi à la fragmentation de ce groupe et aggravent la tension sur les effectifs. Elles offrent toutefois une certaine attractivité au métier, car elles procurent aux CTS l'opportunité de continuer à valoriser leurs savoirs techniques. Néanmoins, et face à la précarité et à l'imprévisibilité que revêt la carrière d'entraîneur au sein des clubs, c'est la procédure du détachement ou de la mise en disponibilité qui est quasi systématiquement utilisée. Ces constats font que la fonction publique d'État reste une valeur refuge ${ }^{90}$. À ce titre, les cas de mobilité externe et plus précisément de démission sont, pour l'heure, exceptionnels.

Alors que les difficultés exprimées par les PTP reflètent surtout les incertitudes dans lesquelles sont plongés le ministère des Sports et ses personnels, de nouveaux et importants chantiers sont engagés. Ils consistent en une revue des missions que l'État garderait à l'issue de la réforme territoriale et interrogent aussi sur la fusion des DRJSCS et la restructuration des DDI afin d'accompagner l'évolution du périmètre des Régions ${ }^{91}$. Consistant notamment à s'interroger sur l'abandon, la réduction ou l'externalisation de certaines prérogatives, ces réflexions ajoutent à l'inconfort qui caractérise toute période de transition.

\footnotetext{
${ }^{89}$ EPSTEIN R., op. cit., p. 229.

90 De Singly François ; Thelot Claude. 1988. Gens du privé, gens du public. La grande différence, Paris, Dunod.

${ }^{91}$ Lire à ce sujet le rapport rédigé par plusieurs Inspections générales ; «L'évolution de l'organisation régionale de l'État consécutive à la nouvelle délimitation des Régions », avril 2015. Le secrétariat général des ministères chargés des affaires sociales a par ailleurs constitué un groupe de travail chargé de décrire les contours des futures DRJSCS et d'examiner les compétences ainsi que les offres de services qualitatives qu'elles seraient à même de proposer.
} 
Quand le mandat s'étiole

\section{Annexe 1 : Présentation de l'enquête}

L'enquête repose sur 47 entretiens effectués auprès de :

- 40 PTP pour lesquels nous avons cherché à varier les fonctions, les lieux et cadres d'exercice (services déconcentrés, établissements, CTS placés auprès de fédérations sportives représentant un large panel de disciplines), les âges et les anciennetés ;

- cinq représentants syndicaux ;

- un expert en droit de la fonction publique ;

- un inspecteur général de la jeunesse et des sports mobilisé dans les diverses missions d'inspection relatives aux personnels et à l'organisation du ministère.

Ces entretiens visaient à aborder les points suivants : 1) Les trajectoires sociales des PTP ; 2) L'explicitation du faisceau de tâches qu'ils effectuent ; 3) Les changements à l'œuvre dans le secteur sportif et leurs effets sur la mise en œuvre de la politique ministérielle ; 4) Le rapport qu'entretiennent les PTP vis-à-vis du rôle qui leur est assigné, de ses éventuelles transformations, plus généralement de leur statut d'agent de la fonction publique d'État; 5) Les formes et enjeux de la mobilisation des organisations syndicales à l'égard du processus de modernisation de l'État.

La retranscription intégrale de ces entretiens et leur analyse par la méthode thématique ont permis que soit livré - au croisement d'éléments objectifs et subjectifs - le sens que les PTP accordent à leur travail et aux diverses mesures adoptées dans le cadre des réformes de modernisation de l'administration. Cette option consistant à apprécier la façon dont les agents eux-mêmes appréhendent leur situation professionnelle, dimension essentielle du rapport au travail ${ }^{92}$, a été réalisée sans méconnaitre la difficulté à cerner la nature de ce rapport à partir de récits subjectifs ${ }^{93}$.

\footnotetext{
92 GOLLAC Michel ; VOLKOFF Serge. 2001. Les conditions de travail, Paris, La Découverte ; LINHART D., op. cit.

93 MEDA Dominique. 2010. "Comment mesurer la valeur accordée au travail ?", Sociologie, $\mathrm{n}^{\circ} 1$, p. 121-140.
} 
Sciences sociales et sport, $n^{\circ}$ 9, 2016, p. 115-152

Alors que les effets des fusions des services et des réductions d'effectifs sur les pratiques de travail se font encore fortement ressentir, la période est également marquée par plusieurs projets de réformes (réforme du périmètre des Régions, projet de loi relatif à la Nouvelle Organisation territoriale de la République, revue des missions de l'État territorial) qui contribuent au caractère profondément instable des processus étudiés. Nous avons ici pleinement intégré cette dimension dynamique dans l'analyse du matériau empirique. Une exploitation de nombreuses sources documentaires a également été réalisée : presse nationale, autres recherches effectuées sur ces objets, bulletins syndicaux, débats au Parlement, rapports publics nationaux, discours ministériels, analyse de contenu des textes (ou projets de textes) législatifs et réglementaires relatifs au cadre d'emploi des PTP et à l'organisation des APS. 